\title{
Mechatronic system for verticalization and aiding the motion of the disabled
}

\author{
D. JASIŃSKA-CHOROMAŃSKA*, K. SZYKIEDANS, J. WIERCIAK, D. KOŁODZIEJ, \\ M. ZACZYK, K. BAGIŃSKI, M. BOJARSKI, and B. KABZIŃSKI
}

Faculty of Mechatronics, Institute of Micromechanics and Photonics, Division of Design of Precision Devices, Warsaw University of Technology, 8 Św. A. Boboli St., 02-525 Warszawa, Poland

\begin{abstract}
Mechatronics is nowadays a dominating concept in design of various kinds of systems and technical devices. High speeds of data processing by control units of mechatronic systems, as well as high dynamics of their actuators allow the systems to be applied in wider fields. This refers to the medicine as well, especially while making attempts to replace lost human motor abilities by means of robots aiding the man. These devices can be divided into three groups: exoskeletons designed to strengthen the natural force of human muscles, orthotic robots that restore lost or weakened functions of human limbs, and prosthetic robots replacing an amputated limb. The first and the third group are known quite well, whereas the orthotic robots are at an initial phase of their development. The authors have worked on a device for aiding the motion of disabled people suffering from paresis of the lower limbs. The paper presents a concept and a structure of the system that has been created, comparing it with similar devices that have already existed. There are indicated some connections and areas where the subunits merge, as well as the rules of their working with the user.
\end{abstract}

Key words: orthotic robots, aiding the human motion, mechatronic systems.

\section{Introduction}

Mechatronic devices and systems, whose microprocessor unit replaces man while making decisions in known situations, are currently a leading group of technical products as far as most of the fields, connected both with manufacturing and everyday life, are concerned [1-28]. They provide also a great opportunity to improve the comfort of life of the people suffering from serious diseases. Besides important applications of mechatronics while building medical equipment adding a treatment of the sick, or even making it possible, there is also a chance to effectively replace the impaired motor activities of the human organism by means of technical devices. Persons suffering from paresis of the lower limbs usually use wheelchairs for moving about. This way of moving about has many advantages, of which the biggest is a sense of security of the disabled person resulting from the stability of the carriage. However, using a wheelchair is also a reason of many significant limitations. A person on a wheelchair sees the surrounding world from a different perspective, as compared to a healthy person. Many elements of the life infrastructure are out of his sight and reach. One of the possible solutions to this problem is so-called verticalization, i.e. setting a disabled person in a vertical position by means of special instruments or devices.

1.1. Methods of verticalization. A man with numb lower limbs can be compared to a mechanical system consisting of an able trunk coupled with a motor system of many degrees of freedom that is void of actuators. A first approach to verticalization of patients consists in immobilizing the joints of the osseous system of their lower limbs by coupling particular sections of the limbs with a rigid skeleton set at a vertical position. The static balance of the verticalized person is ensured by an appropriate arrangement of the points at which the device is supported on the ground. There are a lot of well known technical solutions employed for verticalization of patients that are applied depending on their state of health [1]. These are, among other things (Figs. 1 and 2): verticalizing beds, verticalizing devices - especially: mobile verticalizing tables, verticalizing carriages, and parapodia: static, dynamic, mobile and vertical. Most of these devices and instruments are designed for therapeutic purposes. Verticalization of patients that cannot walk prevents them, among other things, from decalcification of the bones, degeneration of the internal organs, decreasing the heating function of the blood flow and decreasing elasticity of the muscular tissue and ligaments. Some of the instruments can be used for moving the disabled, yet that requires a help of another person or application of a drive (Fig. 1b). An independent moving about of a person suffering from paresis of the lower limbs is possible while using a dynamic parapodium (Fig. 2b, c), however only in the case of flat surfaces, without a possibility of taking obstacles. The motion realized in this way has an unnatural character, since it is realized by balancing with the whole body of the person being verticalized. In order to carry out a rehabilitation of patients that consists in verticalization of their bodies and forcing a motion of the numb limbs, special devices are used, including so-called locomats (Fig. 1c).

*e-mail: danuta@mchtr.pw.edu.pl 
D. Jasińska-Choromańska et al.

a)

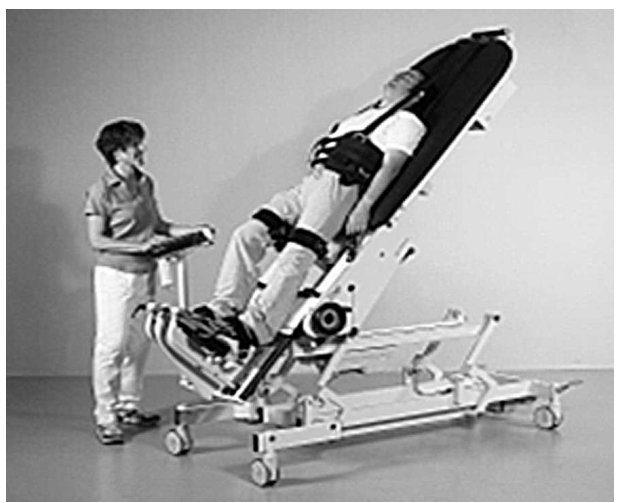

b)

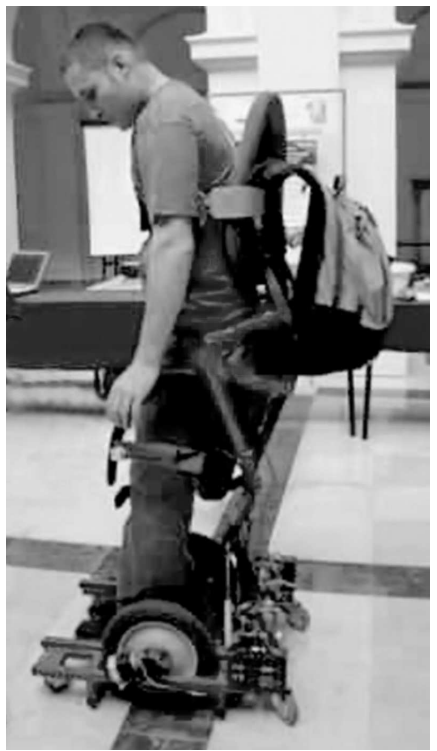

c)

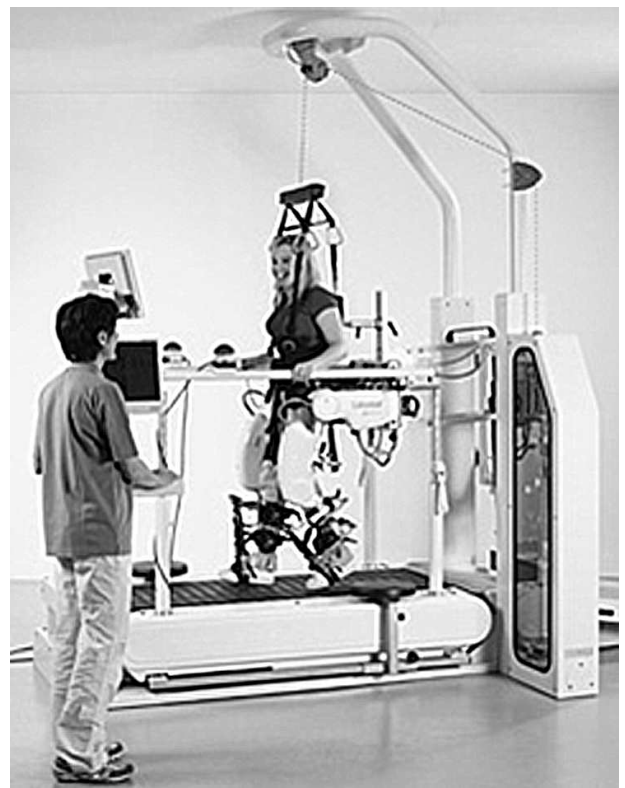

Fig. 1. Devices for verticalization of persons suffering from paresis of the lower limbs: a) dynamic verticalization device [2], b) tetrapodium developed and built at the Faculty of Power and Aeronautical Engineering, Warsaw University of Technology [3], c) locomat [1] a)

b)
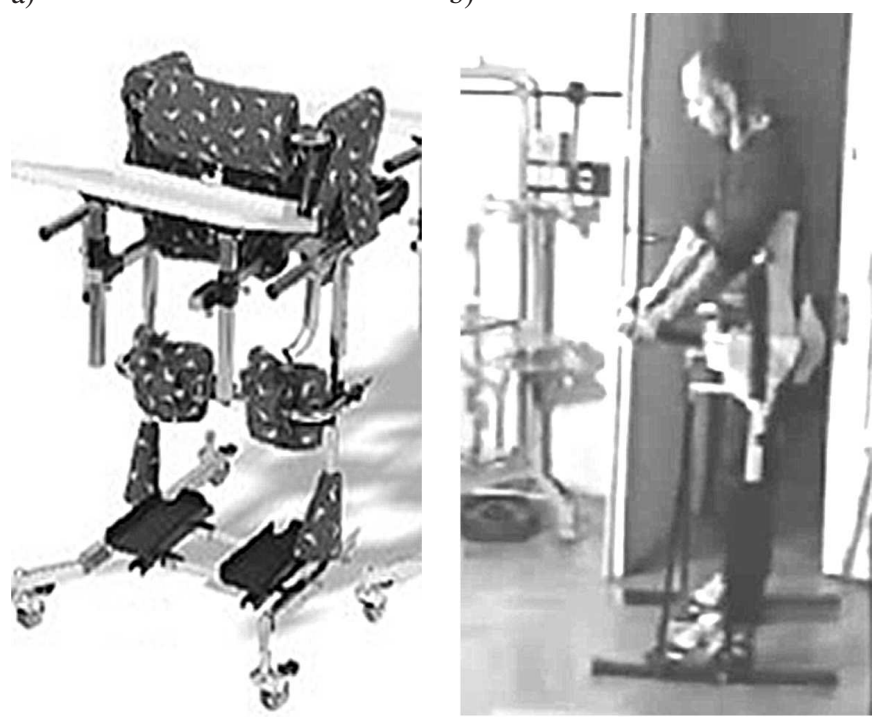

c)

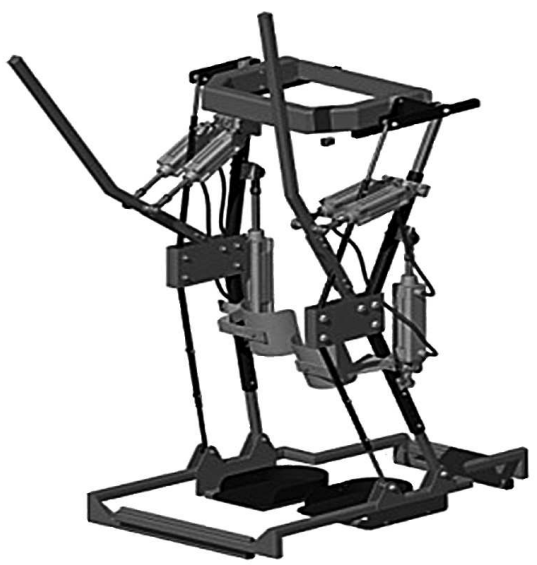

Fig. 2. a) Static parapodium, b) dynamic parapodium developed at the Faculty of Power and Aeronautical Engineering, WUT [3], c) dynamic parapodium developed at the Faculty of Mechatronics, WUT

1.2. Devices for aiding the gait. Progress in the field of systems improving everyday life of persons suffering from paresis of the lower limbs by restoring to them the regular human functioning as much as possible, is strictly connected with a dynamic development of mechatronic systems aiding the natural human movements [6-10]. The systems are called "wearable robots" [6], what is meant to reflect both their technical aspect as well as their close connection with the user (operator), for whom they are some kind of a suit. These robots can be classified into three categories, depending on their relation to the human muscular system [6]:

- empowering robotic exoskeletons - enhancing beyond a standard level the ability of a healthy man, e.g. lifting up heavy objects,

- orthotic robots - used for restoring lost or weakened motor functions of the limbs,

- prosthetic robots - replacing amputated limbs.

Exemplary solutions of robots aiding the motor human functions are presented in Fig. 3. 
a)

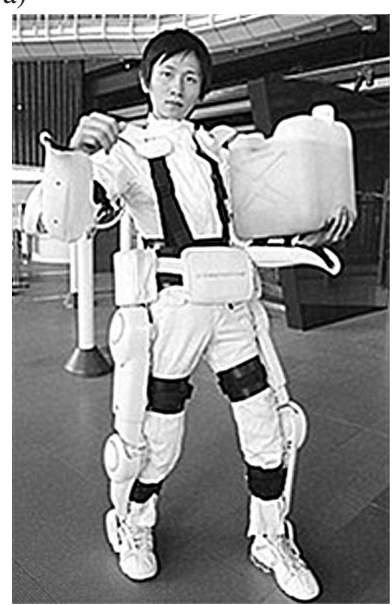

b)

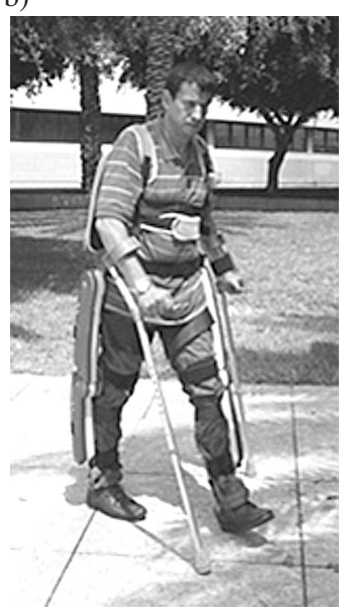

c)

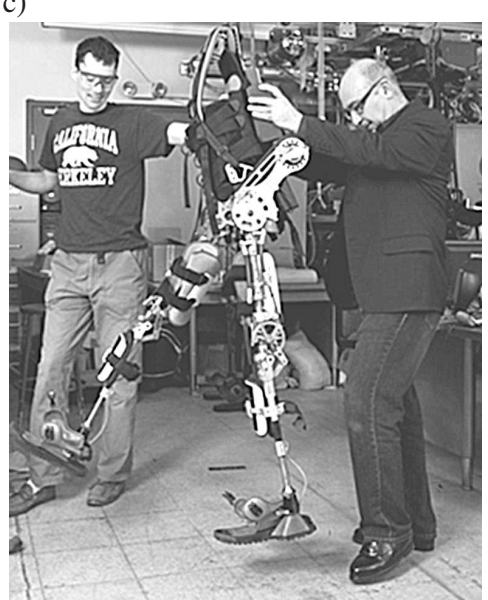

Fig. 3. Examples of robots aiding the human motion activities: a) $H A L$ exoskeleton developed at the a University of Tsukuba, Japan [6], b) ReWalk system realizing gait of individuals suffering from a paralysis of the lower limbs by the Argo Medical Technologies

company [6], c) eLEGS system by the Berkeley University

The robots are used both for verticalization of the patient and forcing his gait. Such systems have been developed at few centers in the world, where they are called "orthotic robots". The pictures present e.g. a device called ReWalk that has been built in Haifa, Israel and is to be commercially available since 2012, as well as the eLegs robot presented recently by the Berkeley University.

\section{System for verticalization and aiding the motion}

The system for verticalization and aiding the motion (SVAM) that has been built (Fig. 4) can be numbered among the orthotic robots. As far as the operational use is concerned, the system has a form of a robot, whose movements are transmitted to the patient's limbs by means of special catches. A backpack contains accumulators supplying the actuators of the device and its controlling computer with electric power. By means of a control panel, the user selects a motion mode that is to be realized according to a program. Additional orthopedic devices allow the user to keep his physical balance.

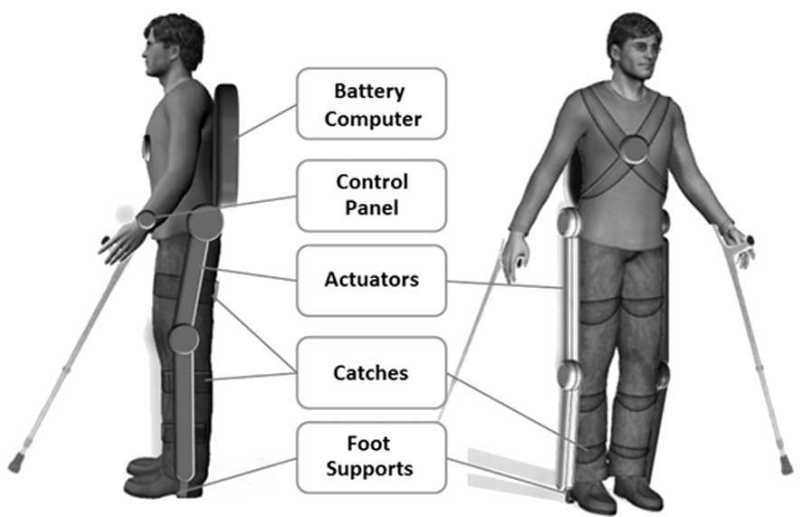

Fig. 4. The system for verticalization and aiding the motion (SVAM)

The main function of the system is to enforce the movement of the lower limbs of the user in a manner similar to a regular human gait. Additionally, the system is to provide the user with the ability of taking some obstacles, including going up and down the regular stairs, as well as sitting on standard furniture and getting up from it. It has been assumed that the device realizes movements of the user's leg according to determined programs. At the present stage of development, the other elements of the gait, i.e. unloading the leg which is shifted and keeping one's dynamic balance is left to the user, who is equipped with additional orthopedic devices, e.g. crutches.

Even though the basic purpose of the device is to restore lost motor functions of the disabled and to reduce the negative medical effects resulting from immobility of the limbs, another important function of the system will be minimization of a risk of additional injuries of the user. The system should eliminate all the possible hazards and prevent realization of any movements if they pose a threat to the patient's health. In the case of the contemporary mechatronic machines, the safety systems are closely integrated with the systems realizing the main function. As emphasized in [10], it is advantageous when both systems use the same actuators and measuring systems, as much as possible. In Fig. 5 there is presented the proposed system of interconnections between particular functional blocks of the robot that has been designed.

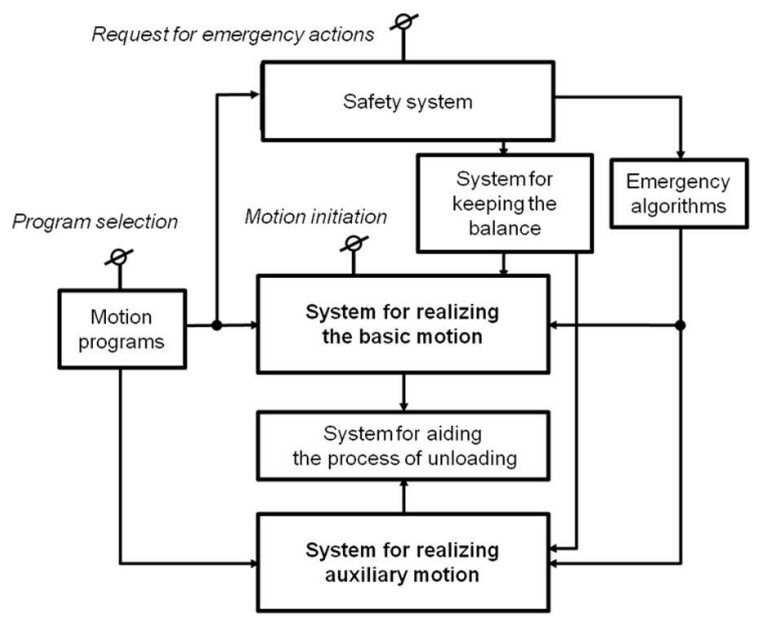

Fig. 5. The proposed functional structure 
D. Jasińska-Choromańska et al.

As robots, devices aiding human movements are typical examples of mechatronic systems, whose structure is presented in a form of a general schematic, as in Fig. 6. Numerous design similarities between the three aforementioned types of robots allow designers originating from various centers to share their experiences they gained while working on various solutions. In the next section, solutions of selected robots aiding the human gait are reviewed, with reference to particular elements of the structure presented in Fig. 6. The review is devoted to the system for realizing the movements, having assumed that this system is the most complicated one in the device and it integrate the remaining systems the most.

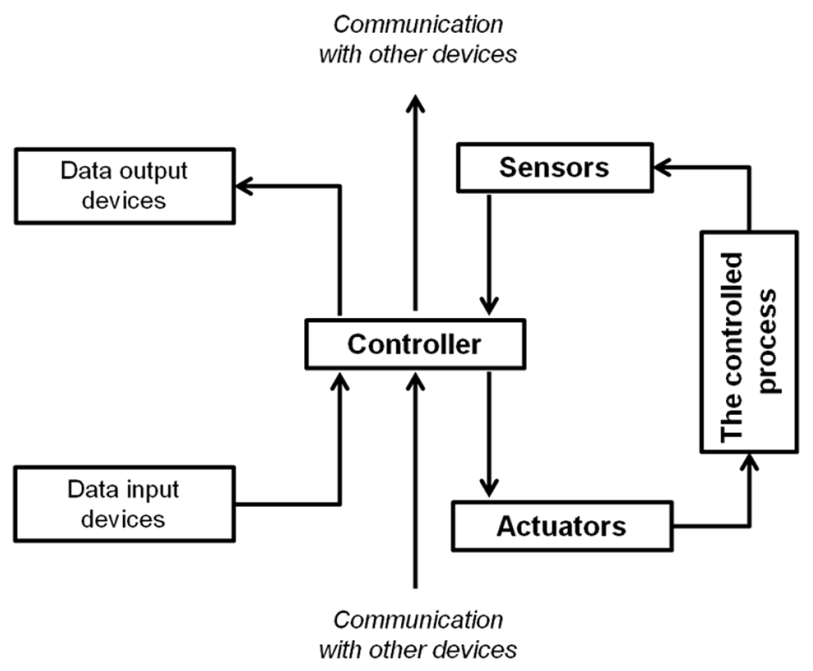

Fig. 6. General schematic of a mechatronic device [11]

2.1. Analysis of the structure of the system with respect to the main function. Identification of particular elements of the system was carried out with reference to the function of forcing the motion.

The control process. According to the accepted assumptions, in the case of the SVAM device, the controlled process consists in putting the numb lower limbs of the user into a motion resembling the natural human gait, or forcing movements corresponding to taking obstacles, standing up or sitting down.

Actuators. Actuators of the device are to be used to move the lower limbs of the user. According to the commonly accepted simplifications [5], each leg has seven degrees of freedom: the hip joint and the ankle joint - each with 3 degrees, and the knee joint - with 1 degree. In the case of the systems aiding the gait, the number of the degrees of freedom is usually limited to 3 degrees for each limb. The driven mechanical members of these robots are fastened to the user's limbs by means of strips that allow the legs to be slightly displaced with respect to the rigid structure of the robot.

In the case of the Japanese exoskeletons HAL-5 (Hybrid Assistive Leg ) [12, 13], designed both for enhancing the capability of the human strength as well as for rehabilitation purposes (Fig. 7), the user's legs are driven by DC motors coupled with harmonic drives, located exactly within the axis of the articulation, and ensuring bending/extension of the hip and the knee. The HAL device, unlike many other exoskeletons, transmits the load to the ground using the human skeletal system, and its only task is to increase torques in the hip, the knee and the ankle joint.

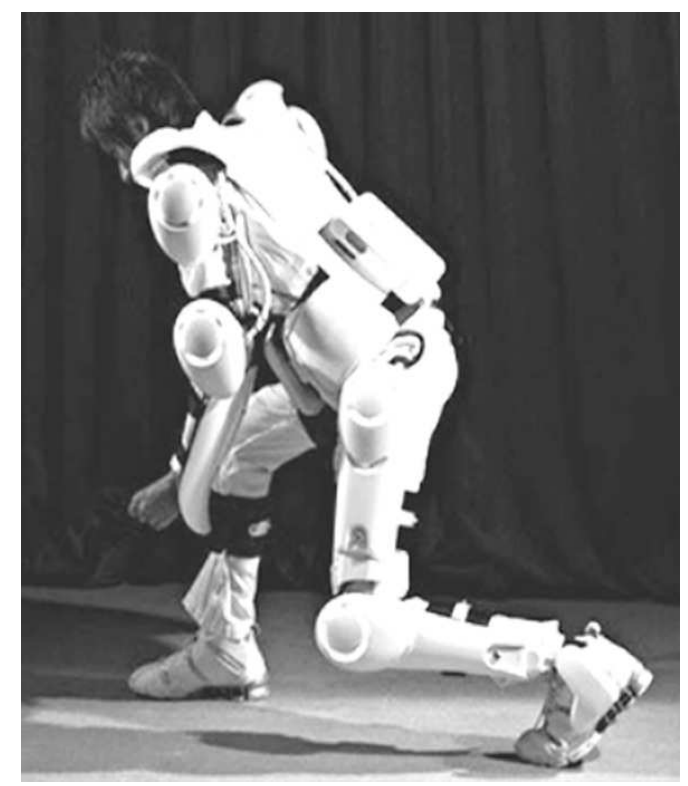

Fig. 7. HAL-5 exoskeleton [13]

The RoboKnee exoskeleton is a relatively simple device for supporting the knee joint while ascending the stairs and squatting [14]. The device operates as a follow-up system, using a special spring-loaded linear actuator (called Series Elastic Actuator) (Fig. 8a) attached to a knee fixator, just below the hip, at the calf (Fig. 8b). The task of the device is to provide an additional torque to the knee at the least physical effort of the user. The control system of the RoboKnee device employs force sensors measuring a reaction of the ground in a vertical direction and sensors locating the center of gravity within the fibular plane. This information is used by a positive feedback system whose task is to increase the torque in the knee. However, it can measure neither the lateral forces nor the angle of the force vector with respect to the ground. The bending angle of the knee is determined by the position of the actuator, which is measured by a linear incremental sensor.

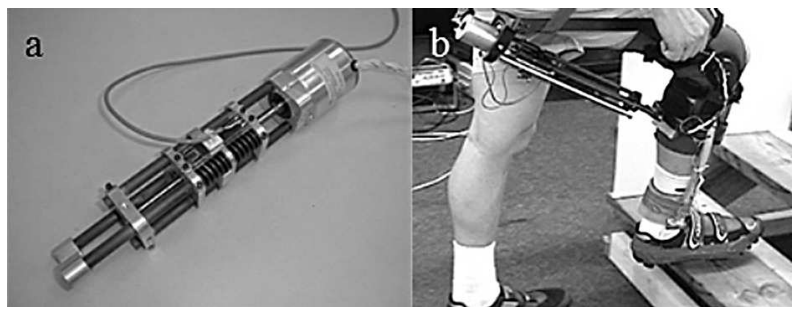

Fig. 8. a) Spring-loaded linear actuator, b) application example of the RoboKnee exoskeleton [13]

The Sarcos exoskeleton [15] supports the muscular systems of the whole body of the user (Fig. 9). It is supplied by a portable energy source. It employs hydraulic rotary actuators located exactly at the driven articulations of the device. 


\section{Mechatronic system for verticalization and aiding the motion of the disabled}

For the control purposes, the Sarcos senses the force of the interactions between the user and the robot, what is meant to recognize the user's movements and to adapt to them the movements of the exoskeleton ("get out of the way" type of control). The control unit of exoskeleton processes measuring signals form force sensors attached to a metal plate, on which the user puts his foot. As the plate is rigid, it prevents the foot from bending.
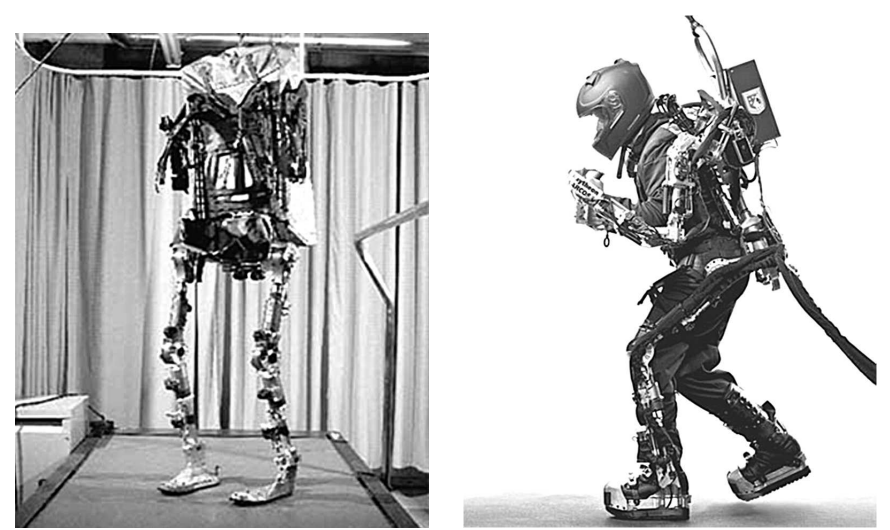

Fig. 9. Sarcos exoskeleton and a user empowered by this device [15]

Currently, in the terms of preparation for a widespread use, the most advanced exoskeleton of the lower limbs designed exclusively for the disabled is a ReWalk device designed at Argo Medical Technologies Company. The robot is equipped with electric drives. Several possible solutions of systems that drive the robot joints are presented in Fig. 10.

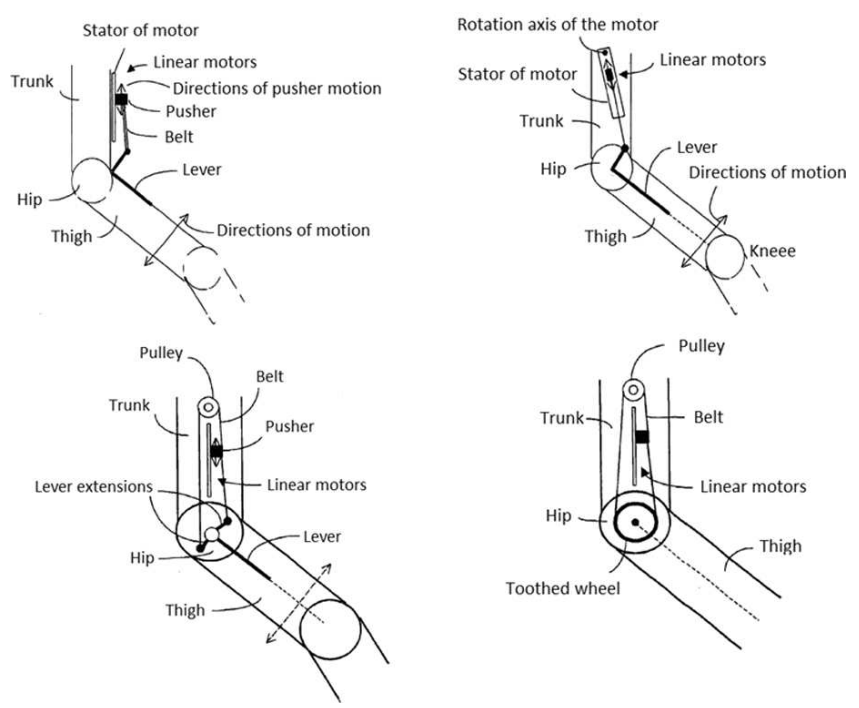

Fig. 10. Design variants of the drive systems for moving the legs, according to the patent related to the ReWalk robot [16]

Measuring systems. In the case of exoskeletons controlled on the basis of the signals generated by the human nervous system, their sensory systems are highly sophisticated, both in terms of hardware and software. For instance, the control system of the $H A L$ robot uses EMG electrodes placed on the skin surface below the hip and over the knee, and additionally force sensors measuring reaction of the ground. A gyroscope and an accelerometer mounted in a backpack are used to determine an instantaneous tilt of the user's trunk. The sensors work with two control systems: one based on the EMG signals, and the other based on gait profiles. The first recognizes the user's intentions, whereas the second controls the actuators [13].

In the case of the device that has been designed, due to the accepted concept of realizing the movements of the limbs according to a program, the measuring systems will be used to verify correctness of the movements being realized, and in particular to verify their execution (completion). Much greater role will be played by the transducers generating signals to the safety system and the system of balance keeping.

Input devices. As far as the orthotic robot is concerned, the input devices are used to:

- input data necessary to start the system,

- select a specific operation program and its parameters,

- initiate movements.

In the case of the ReWalk device [17], a basic input device for the user is a special control panel (remote control) attached to the wrist, equipped with buttons for selecting appropriate operation mode (Fig. 11). To initiate movements, there is employed an accelerometer fastened to the user's trunk.

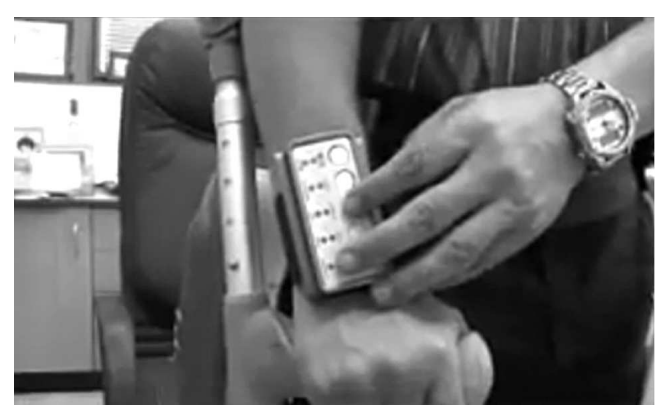

Fig. 11. Control panel of the ReWalk device [17]

Output devices. The task of the output devices is notifying the user about any relevant fact that might affect operation of the system. In the case of the system for moving the legs, such information is related to:

- possible faults detected in the system by the diagnostic units,

- current level of the auxiliary energy in the portable power source.

A poll taken among the disabled did not give an unequivocal answer to the question about the expected manner of informing the users. Therefore, it can be planned to adapt this manner to individual needs, i.e. to design and interchangeably use video and audio devices, or devices of other kind.

\section{Results}

3.1. Analysis of the human gait . Orthotic robot, as a sophisticated mechatronic system, requires solving many problems of a technical character, as well as adapting it to working with 


\section{Jasińska-Choromańska et al.}

a live human organism. While starting the works on this system, it was planned to use results of studies and analyses of human motion obtained in previous projects. They became a basis for creating simulation models aiding the design works on the system. The studies concerned movements of the lower limbs at three joints: hip, knee and ankle joint (Fig. 12).

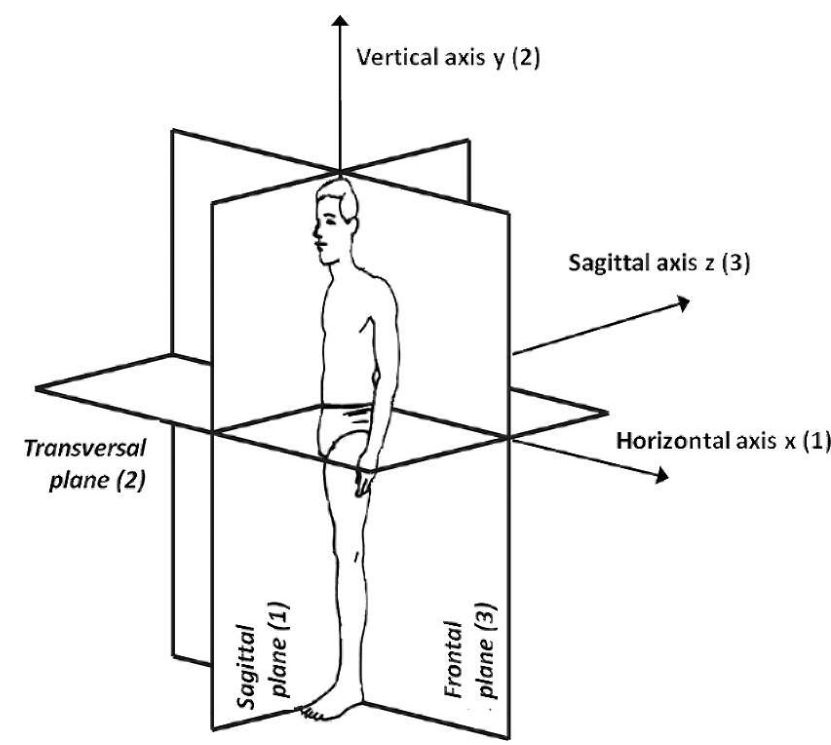

Fig. 12. Measurements of the angle changes over a time for three joints of the lower limbs: hip joint, knee joint, ankle joint (measure-

ments by a motion within a single plane - the sagittal plane)

At first, results of the studies related to walking on a flat horizontal plane were used (Fig. 13a). Because of the accepted operational concept of the device, measurements of the gait when the examined person is supported with crutches are of special significance. There were also used motion profiles while sitting down and standing up, when the examined person was supported with crutches. It is foreseen that the system will allow the disabled to use stairs. Therefore, the analyses pertaining to the motion included also movements that accompany going up and down the regular stairs (Fig. 13b). The motion is supported by crutches. a)

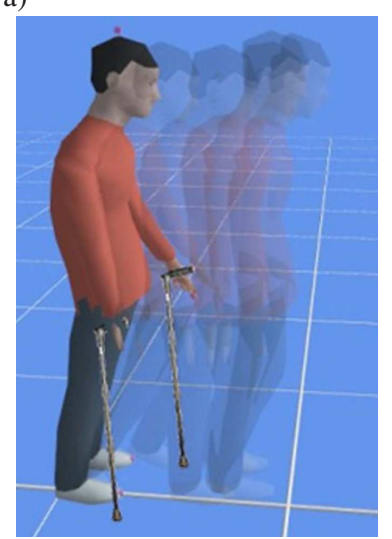

b)

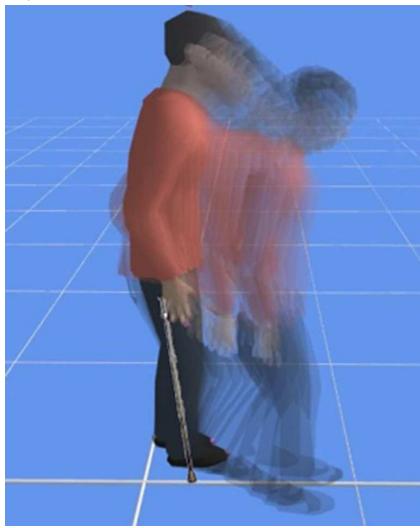

Fig. 13. Visualization of the movements a) walking on a flat surface, b) going down the stairs
At the graphs (Fig. 14), there are presented time courses of the bending angles in particular joints for ten selected cases. On the basis of this data, averaged characteristics were introduced and used while modeling the system that has been designed.

a)

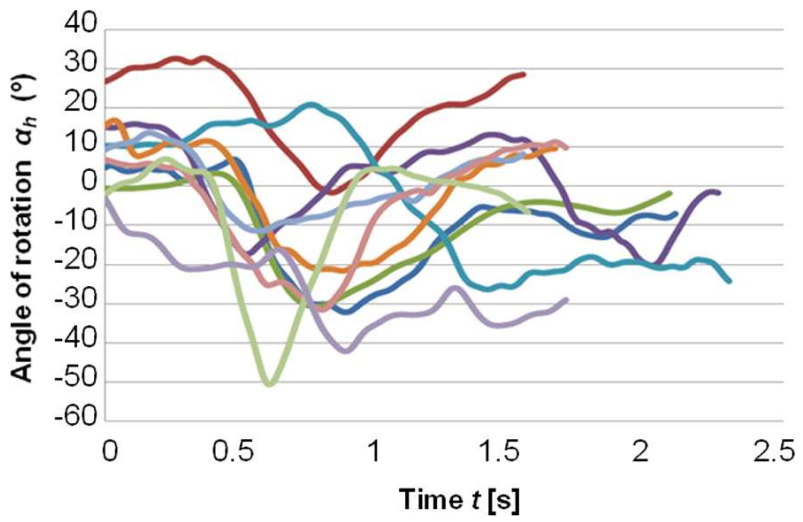

b)

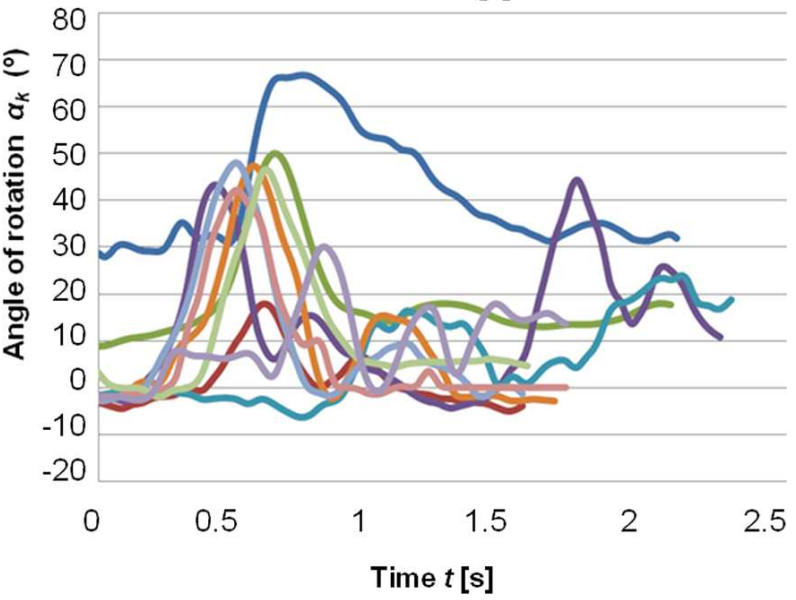

Fig. 14. Time courses of the rotation angles in particular joints for ten selected cases

Functions of the load-bearing structure. The task of the SVAM is to make it possible for a person with impaired motor functions of the legs to keep a vertical position, to walk using the system as well as to stand up and sit down. Users of the system can be both a person that lost the use of his legs (the device realizes then programmed gait functions), as well as a person whose gait is only aided. In both cases, the load-bearing structure of the SVAM realizes a function of a manipulator displacing the user's legs according to the preset function. In the case when the system takes over performance of the gait, the motion is realized according to a preset trajectory reconstructing the gait of a person with certain anthropometric parameters that have been preset. In the case when an aided gait is to be realized, the system operates as a follow-up system with a position or force feedback.

The basic function of the load-bearing structure is realization of motion of the limbs reconstructing the kinematics of the user's motor organs as precisely as possible. The motion is realized by transmitting movements of the actuators to the user's limbs by means of the elements of the load-bearing structure. 


\section{Mechatronic system for verticalization and aiding the motion of the disabled}

The mechanical structure of both versions of the system, i.e. aiding the gait and realizing it fully, should be the same. Additional functions of the load-bearing structure are:

- Transmitting the weight of the device to the ground,

- Transmitting (fully or partially) the weight of the user to the ground,

- Keeping the user inside the structure of the SVAM,

- Providing a fastening for other elements of the SVAM.

Determination of the kinematics. Literature pertaining to the theory of design of machines and mechanisms [18] and to the walking machines [19-21] considers the mechanical system reconstructing the human gait as an open system a manipulator. As far as this definition is true with respect to walking machines, in the case of the SVAM another approach should be tried. If foot 5 (Fig. 15) were considered an actuator, and joints 2, 3 and 4 were reduced to kinematic pairs of $5^{\text {th }}$ grade, then the limb could be considered a double pendulum manipulator with members $a, b$ and frame 1 . In the case when the kinematic chain $2-a-3-b-4$ is used for shifting the user's foot (Fig. 15 on the right), placed within the system foot 4, a change of the kinematic structure will take place. The open kinematic chain 2-a-3-b-4 is connected with a chain 4-d-3'-c-2' and thus a closed system is created. Axes of articulations 2 and 2' as well as 3 and 3' may overlap with the accuracy corresponding to the fastening of the system 2-a-3-b-4 to the user's body. So, the real kinematic configuration will be a five-bar linkage, where the kinematic chain of the user's limbs imposes kinetic limitations on the system. Because of the necessity of ensuring security for the user, these limitations should also exist in the chain that is created by the elements of the SVAM.
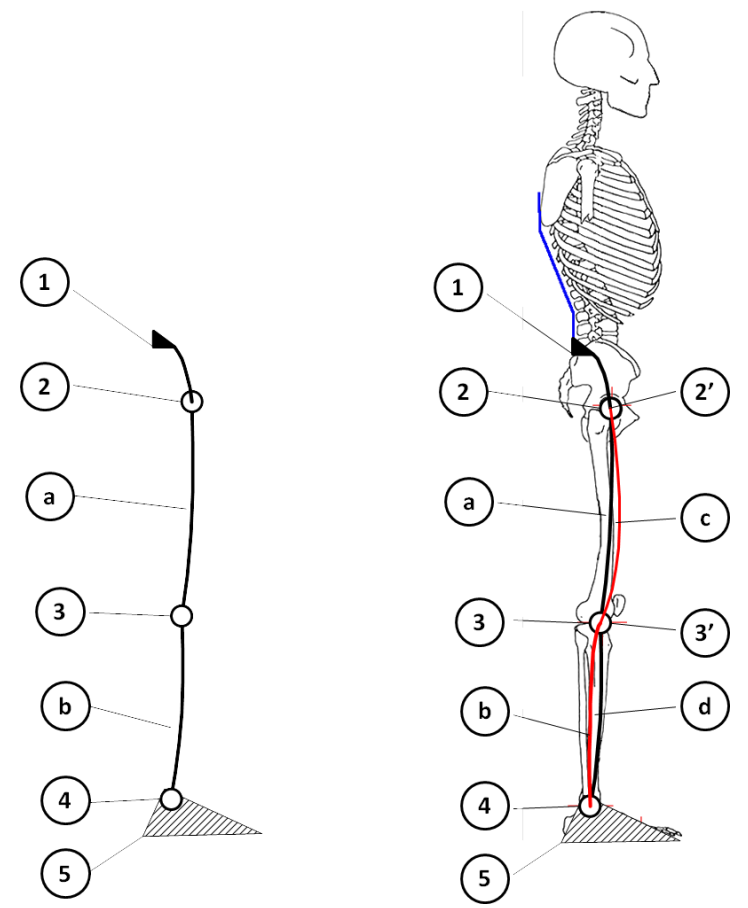

Fig. 15. Kinematic diagram of the limb considered an open system (on the left) and a system with closed kinematics (on the right)
Assumptions regarding the mechanical structure of the SVAM. Having discussed and analyzed the kinematics, it was assumed that the considered mechanical structure of the SVAM will be regarded a mechanism that was presented above and is a merger of the active kinematic chain of the device and the passive chain created by the human motor system. The assumption results also from the fact that shifting a free (impaired) human leg can be realized by running the heel along a preset trajectory.

Kinematic configuration of the load-bearing structure. Analysis of the human gait proves that the motion in the knee and ankle joint is realized in fact within the fibular plane. Angular displacements about the vertical axis (so-called long axis) are negligibly small and can be compensated for by a motion at the spots where the SVAM is fastened to the user. In order to ensure security of a user with impaired legs, a potential turn in the ankle joint about the fibular axis must be prevented from. That implies that a turn in the ankle joint can be realized by a turn about a lateral axis only. The simplest kinematic structure of the SVAM, assuming elasticity of the contacts between the system and the user, can be therefore realized as a configuration with articulations of the $5^{\text {th }}$ grade (uniaxial) (Fig. 16).

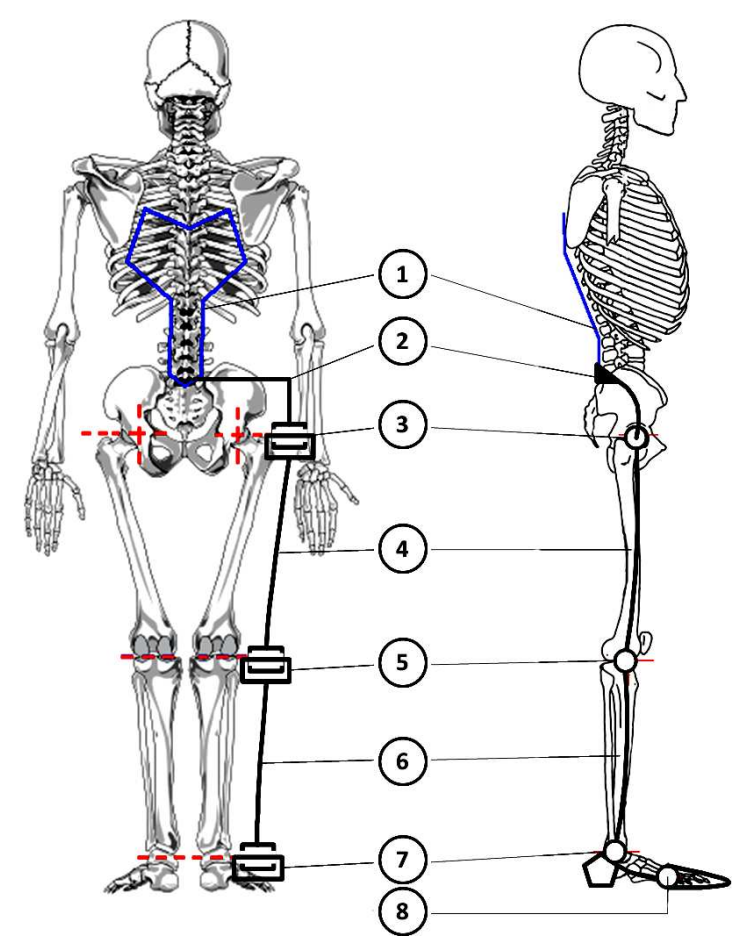

Fig. 16. Kinematic scheme of the SVAM with uniaxial articulations (description in the text)

As far as the above structure is concerned, element 1 is the frame and it is a support for the user's spine within the pectoral and lumbar-sacral region. Hip articulation 3 is fastened by means of coupler 2 . The successive couplers 4 and 6 connect knee articulation 5 and the foot system with ankle articulation 7 and toes articulation 8. For the sake of simplicity, the foot system is shown as without springs. This is the simplest design solution, however because of safety reasons 
D. Jasińska-Choromańska et al.

and the user's comfort, it can be employed only in a limited range, i.e. in a system used for aiding a person that can walk by himself.

In the case of the hip joint, during a correct gait there occurs a turn about an axis that is perpendicular to the human lateral axis (bending and extension), a turn about the vertical axis (pronation and supination) as well as a turn about the fibular axis (abduction and adduction of the thigh) resulting from a possible balance keeping, yet in a small extent. While assuming that the function of balance keeping is not fulfilled by the system but by the user walking on crutches, abduction and adduction of the thigh while walking can be omitted in the further analysis. However, these movements are necessary while e.g. walking sidelong and apposing one leg next the other after standing up. Performing this motion will be also advantageous, assuming an identical structure for the version aiding the gait and realizing it artificially. Two possible solutions of the kinematics with abduction and adduction of the thigh are presented in Fig. 17.

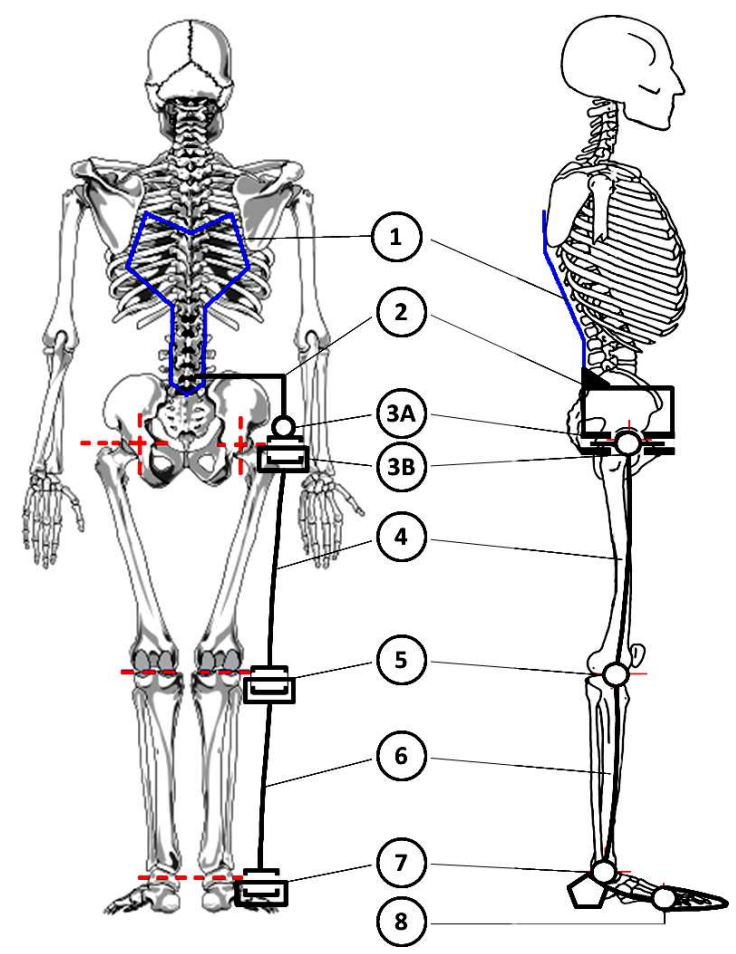

Fig. 17. Kinematic diagram of the SVAM with uniaxial articulations, featuring an extra possibility of abducting the leg within the hip joint (description in the text)

If articulation $3 \mathrm{~A}$ connected with articulation $3 \mathrm{~B}$ were added to the configuration presented in Fig. 17 the leg could be abducted. Because the axis of articulation 3A does not overlap the axis of the hip joint, adduction and abduction will be possible within a smaller range compare to their anatomic counterparts. An advantage of this solution is displacing the actuator away from the body, however it cannot occupy too much space because of the comfort of the user. This solution, as the previous two, suits more the case of aiding the gait of a person that can walk by himself.
The second of the elaborated diagrams (Fig. 18) assumes introduction of a revolution axis of an additional articulation in the axis of the hip joint. Because of physiological reasons, such system cannot stick out of the outline of the pelvis, and cannot be located at the front.

A configuration with a four-bar linkage $2 \mathrm{~A}$ has been proposed, where one of the members is a linear actuator $2 \mathrm{~B}$ changing the length of the respective bar. Owing to this solution, fastening $3 \mathrm{~A}$ can turn about the anatomic axis supporting articulation axis $3 \mathrm{~B}$ that pierces the central point of the hip joint. In Fig. 18 there is also presented a double-side fastening of the axis of the ankle joint 7 and application of a six-membered mechanism in the knee joint. That solution meets the safety requirement referring to the system realizing a forced gait, however, because of the reasons discussed later in the text, it features some limitations, as far as correctness of the gait is concerned.

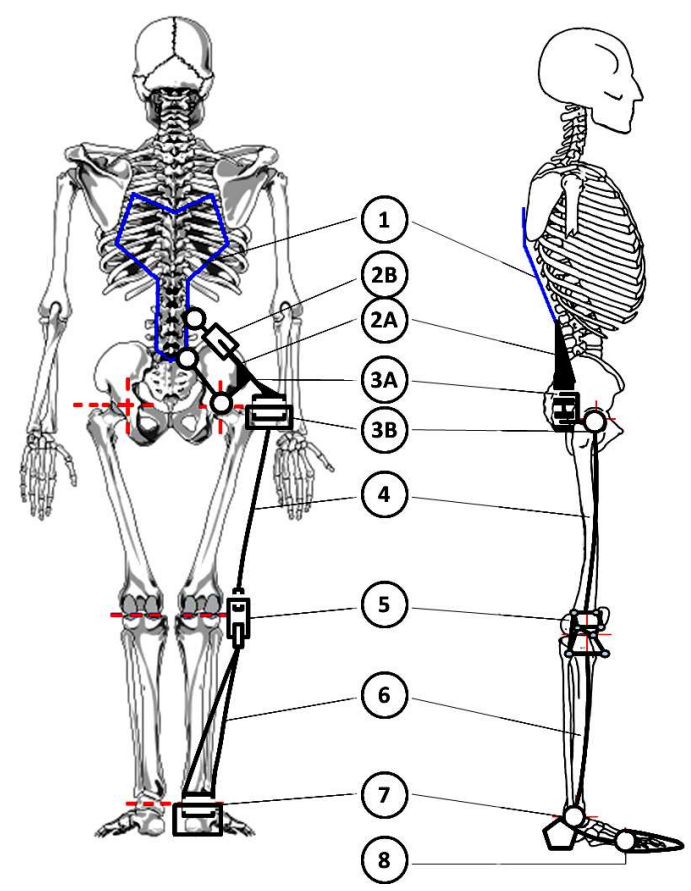

Fig. 18. Kinematic diagram of the SVAM with a four-bar linkage in the hip joint and a Stevenson mechanism in the knee joint (description in the text)

One of the limitations is a lack of possibility of turning the pelvis about the vertical axis while stepping forward, provided the foot does not turn with respect to the ground. Turns of the pelvis (Fig. 19) are necessary in order to ensure that the feet are put on the ground in a steady way, and ensure their stable position while transmitting the load from one leg to the other [22]. It also allows the swing of the leg to be lengthened and the kinematic energy of the trunk to be used, decreasing thus the energy consumption. If such motion were possible, it would be advantageous for the user and it would improve the comfort of walking. 


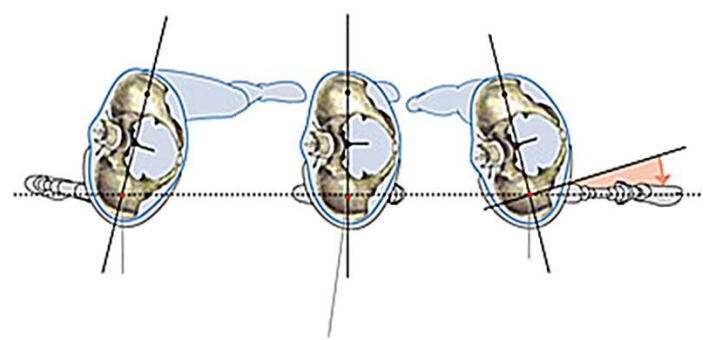

Fig. 19. Turns of the main plane during the gait realized by a turn of the pelvis with respect to the motion plane [22]

The above postulate can be realized employing the following kinematic diagram of the SVAM.

This configuration is a modification of the configuration presented in Fig. 18. An additional articulation 3C has been introduced, located between articulation 3B and coupler 4. It operates in an axis that is perpendicular to the vertical axis of the joint. The motion in this articulation should be limited to values resulting from pronation and supination in the hip joint while walking. Forcing the motion, as in the case of the ankle joint, can be realized in a passive way using the motion energy of other elements of the system. Realization of this solution, even though it is the most complicated one, would be more innovative since none of the existing solutions of the systems aiding the motion reconstructs so many degrees of freedom related to the human motion.

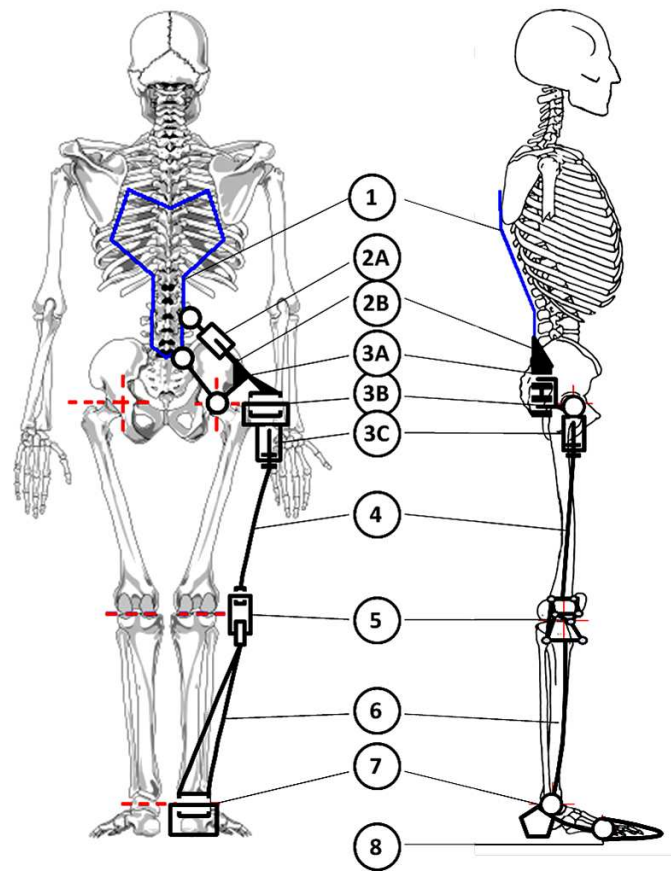

Fig. 20. Kinematic diagram of the SVAM with a four-bar linkage in the hip joint and a possible turn about the vertical axis, and a Stevenson mechanism in the knee joint

The mechanical structure of the system is one of the most difficult design tasks. Recently, a first version of the structure has been designed. The system that has been designed will contain a limited number of degrees of freedom compare to the human kinematics. A close connection of the mechanism with man will take place within the region of the back and the heel. Many possibilities of building the mechanical system were considered, starting with the simplest ones to very complicated ones. It was decided to build the simplest solution first, yet ensuring a possibility of a further development. Special attention was paid to the movement of the foot while walking. The appropriate reconstruction ensures security and comfort of walking for the person using the system (Fig. 21).

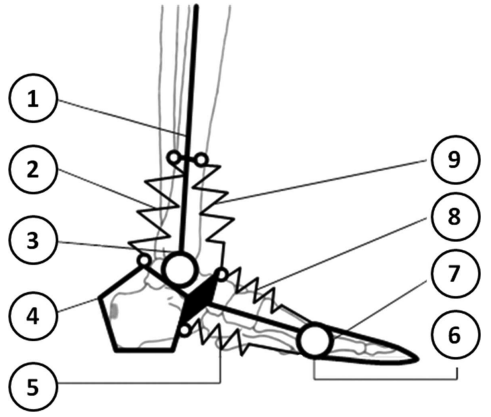

Fig. 21. The analyzed foot model. A mutual movement of element 1 and the grip of the heel and the metatarsus 4 results in compression of spring 2 and 9 and thus putting them under tension. This corresponds to the anatomic sole and spinal bending at a flat foot. The toes rest in the frontal part of the system foot 7 that is coupled with grip 7 by means of articulation 6 . Bending the toes results in putting spring 5 under tension, and thus accumulating energy that is needed for taking-off. Spring 8 ensures return of the toes to the initial position

Another important joint whose reconstruction is crucial, is the knee joint (Fig. 22). Accuracy of reconstructing the motion of this joint conditions ensures the comfort of walking and consistency of the motion kinematics. The best effects are obtained while using six-link mechanisms.
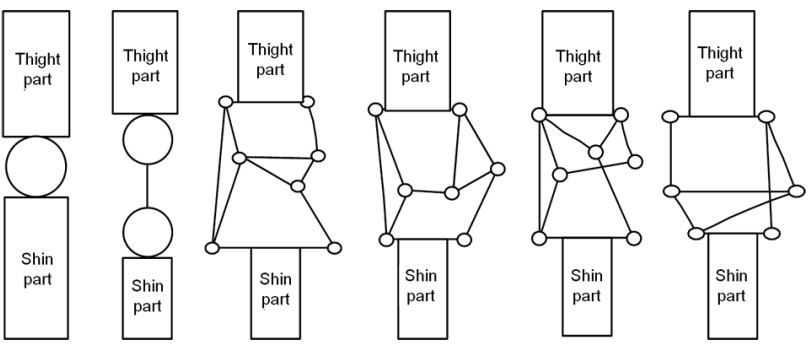

Fig. 22. Model of the analyzed knee joint

3.2. Control system of the SVAM. Because the system that has been built is of a special kind, a problem of ensuring security of the user is superior with respect to its main function. In order to realize this assumption, it was accepted that the control system of the device that has been designed is to make it a self-optimizing system [23]. So, the control system has been created in such a way that it aims at an appropriate objective of action of the device, depending on the situation when the user finds himself in:

- launching, realization and supervision of the motor functions, provided the conditions ensure a safe moving for the patient.

- protecting user's health and life, should a hazard occur. 


\section{Jasińska-Choromańska et al.}

The block diagram of a control system that meets the above assumptions is shown in Fig. 23. The modern approach to development of automation systems called Concurrent Model Driven Automation Engineering (CMDAE) assumes exchange of models between engineering tools used by various discipline engineers [24]. In the device under design the motion programs are developed on the basis of model gait profiles developed upon experiments. The profiles are angular displacements in particular joints in function of a so-called relative normalized time, which takes the value of $100 \%$ for the full gait cycle. The cycle includes a phase of support, when the limb transmits a load generated by the trunk, and a phase of swing, when the limb is unloaded and shifted in the direction of motion. Experimental profiles were approximated by means of Fourier series, taking thus a form of a cyclic function, and then they were used as set values for controlling the drives (Fig. 24).

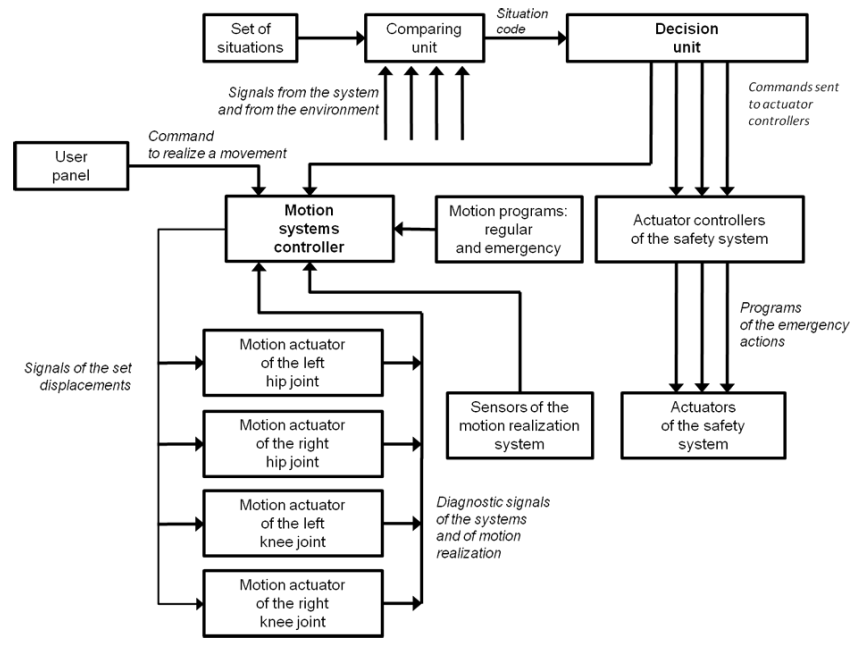

Fig. 23. Block diagram of the control system

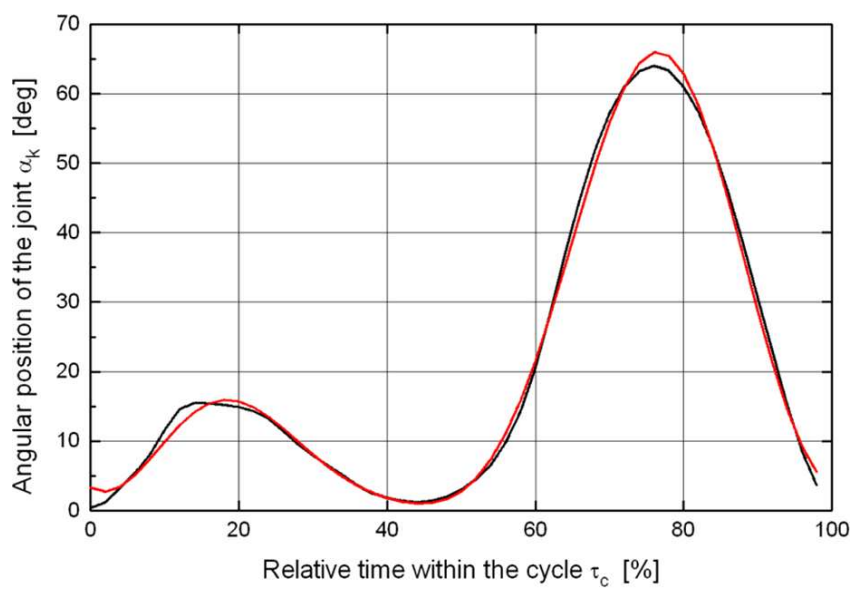

Fig. 24. Exemplary motion profile in a joint: motion of the knee joint while walking on a flat surface and its approximation

The system has a modular structure (Fig. 25), which simplifies any further modifications and development of the device, especially including additional measuring systems. Modules related to the mechanical sub-system of the device and the battery are interconnected by the supply bus and the communication bus. External modules, i.e. the remote control and controllers processing the measuring signals related to the crutches, have their own battery supply and employ radio communication with the central unit.

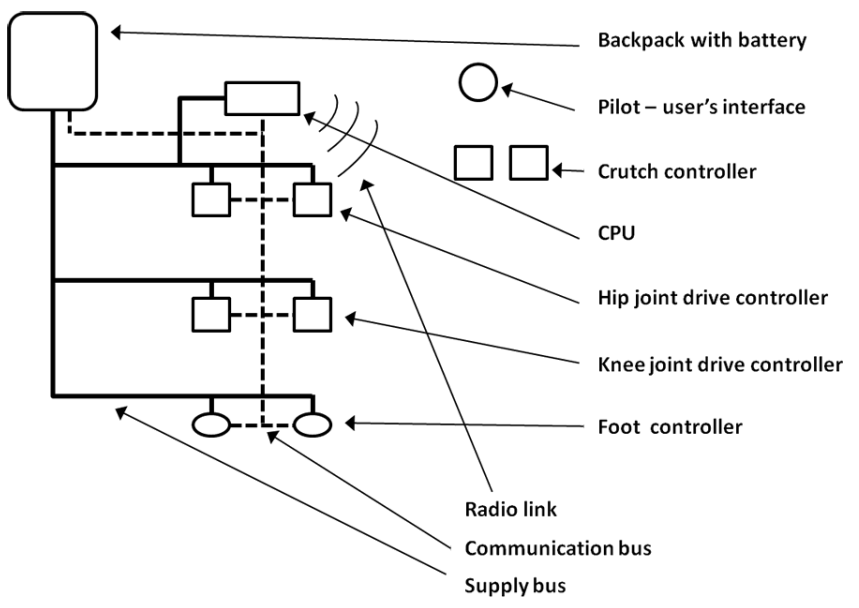

Fig. 25. Modules of the control system

The central unit performs a superior function with respect to the other modules. By means of the communication bus it sends control commands and supervises operation of the whole system. On the basis of the data from particular modules, it detects emergency states. The unit is equipped with a memory slot, what makes it possible to record data while the system is operated. It allows correctness of the operation to be analyzed and the control algorithms to be optimized. The unit can be connected to a $P C$ via the $U S B$ port. Owing to that, it is possible to control the whole system by means of a computer, what simplifies prototyping the control algorithms. The unit is also equipped with a module of position sensors, including among other things, accelerometers and gyroscopes.

The foot controller makes it possible to perform a multipoint measurement of the pressure of the foot against the ground as well as measurement of the bending angle of the ankle joint. The measurements will be used for detection of the phase of motion and prediction of the operator's intention. The system makes it also possible to connect two ultrasonic distance sensors, in order to detect obstacles and stairs.

The battery is placed in the backpack, which allows cells of relatively large dimensions to be used, thus guaranteeing a long operational time of the device. Such solution is useful especially at the phase of testing the device. It is planned to use $\mathrm{LiFePO} 4$ batteries because of high density of energy as well as a high level of security. LiPo batteries are also taken into consideration because of their lower dimensions and weight. The battery is integrated with a special control system ensuring its proper maintenance.

3.3. Simulation studies of the system. Model studies are an inherent part of design works on mechatronic devices and 


\section{Mechatronic system for verticalization and aiding the motion of the disabled}

systems $[25,26]$. Owing to them, it is possible to optimize mechanical structures, electronic units and control algorithms, eliminating costly laboratory tests on real technical models. When designing autonomous walking machines one of essential problems is to assure the highest possible energy efficiency of locomotion [27]. In the case of the system that has been designed, the most important criteria for evaluating the design with regard to its performance and operation, are:

- ability to realize the required motion profiles in the driven joints,

- low energy consumption because of the portable supply source.

Among all the elements of the system, the most significant influence on both criteria listed above have actuators used for driving particular joints (Fig. 26). Thus, first simulation studies were carried out on a model of the systems responsible for realization of the movements. With accordance to the proposed design solutions, the following assumptions related to the model were accepted:

- all the joints are driven only within the fibular plane, thus each lower limb has three degrees of freedom.

- the driven mechanisms have a form of cylindrical articulations,

- the mechanisms are driven by DC motors coupled with reduction gears,

- reference signals for particular units are approximated, averaged motion profiles, determined for healthy individuals.

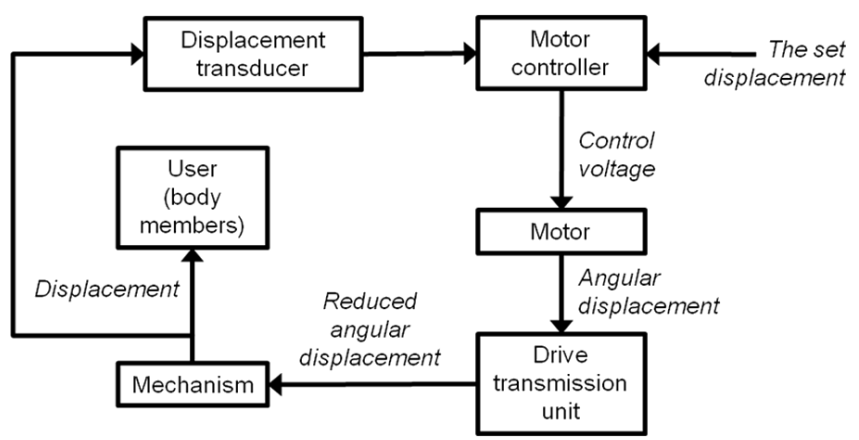

Fig. 26. Diagram of the actuator realizing the motion

Additionally, for the sake of simplicity, the following assumptions were accepted:

- body members of the user are rigid solids,

- frictional resistance at the user's joints is negligibly small,

- a phase of a double-support does not occur during the gait.

Structure of the simulation model includes (Fig. 27):

- motion profiles in the joints, given as the input for the drives,

- drive units ensuring motion of the orthotic robot,

- model of the robot that forces motion of the user due to its connection with the model of the user's body,

- model of the user's body that together with the robot model constitutes a load for the drives.

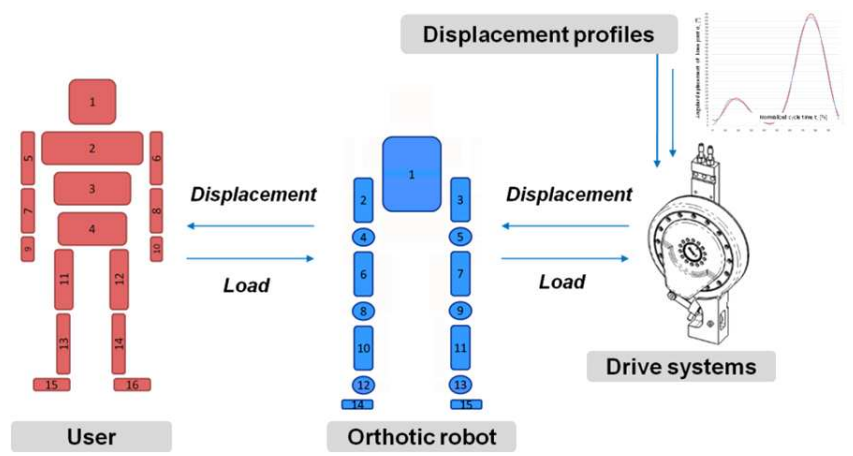

Fig. 27. Structure of the simulation model

The simulation model was created using MatlabSimMechanics software [28] designed for modeling mechanical systems. The parameters that were introduced in the model allow some studies to be carried out, taking into consideration individual features of the user, including its height and weight. Couplers of the robot automatically adjust their length to a declared height of the man. This makes it possible to evaluate influence of an increase of the height and weight of the user on the power demand of the system, precision of reconstructing desired motion profiles and maximal torques that must be transmitted by the articulations and the drive units. In Fig. 28, there is presented influence of the gait rate on the energy consumed by the system. This dependence indicates a significant share of dynamic phenomena in energy consumption by the actuators. For shorter gait cycles, accelerating and decelerating the driven body members results in a significant increase of the energy consumption. One studied also influence of chosen design features of the actuators both on the energy consumption as well as fidelity of reconstructing the desired motion profiles, that is evaluated by a maximal instantaneous error of the angular displacement in the articulation. Graphs presented in Fig. 29 illustrate a possibility of optimizing the ratio of the gear with respect to the power demand of the system as well as its ability to realize the desired motion profiles.

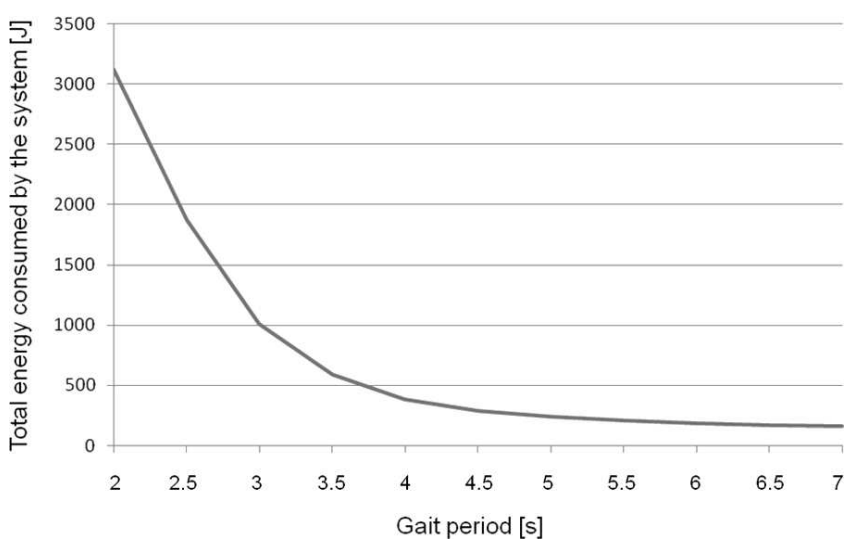

Fig. 28. Graph illustrating dependence of the energy consumed by the system during a single gait period on the length of the period 
D. Jasińska-Choromańska et al.

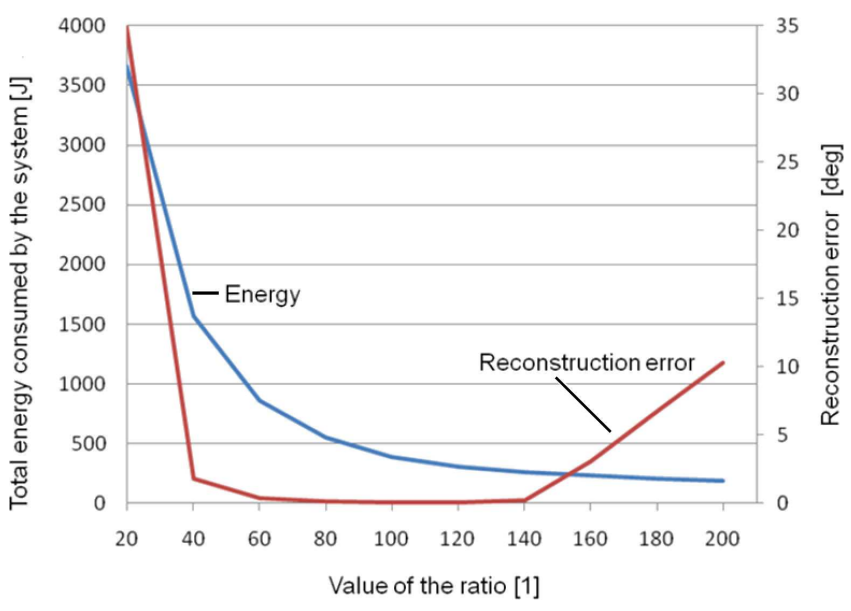

Fig. 29. Graph illustrating the energy consumed by the system and the error of reconstructing the gait profile in function of the value of the ratio for a gear with efficiency of 0.5

The simulation program makes it possible to calculate an instantaneous torque demand of each driving unit (Fig. 30). It is an essential information while selecting the driving motors because of the fact that application of a motor featuring a stall torque too low to make realization of the required movements possible, and on the other hand, application of a motor that is too powerful, resulting in an excessive increase of the mass of the mechanical structure of the device, which is disadvantageous because of many reasons, both technical and functional.

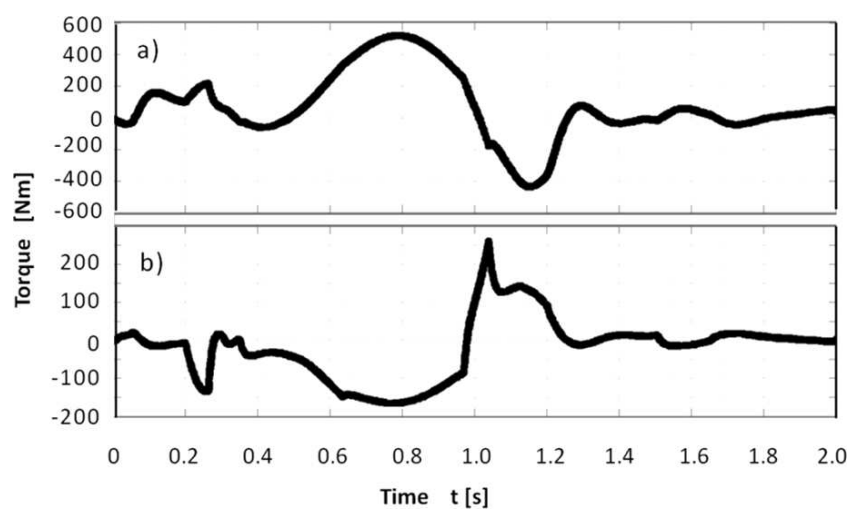

Fig. 30. Examples of the torque courses: a) at the hip articulation, b) at the knee articulation

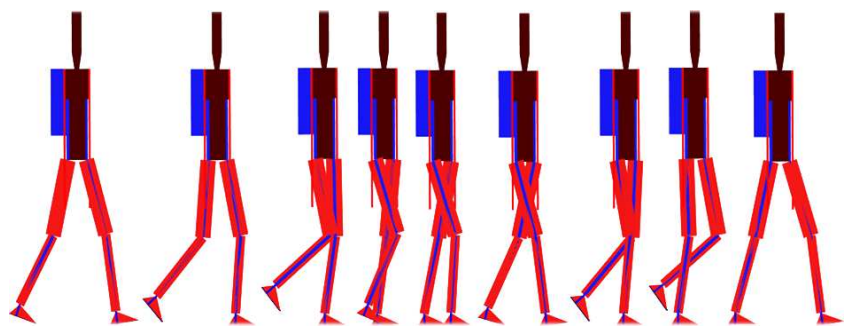

Fig. 31. Picture illustrating operation of the simulation program

A simulation model that has been additionally created makes it possible to visualize behavior of the orthotic robotman system during the gait. In Fig. 31, there are shown po- sitions of the robot members in the successive moments of a single gait cycle. The blue color represents elements of the robot model, whereas the red color (and the brown) represent elements of the model of the user's body (colors in an on-line version).

Results of the simulation studies were used while creating a design of a technical model of the device.

\section{Building a technical model of the SVAM}

While creating the mechanical design, it was decided to use a modular structure of the system (Fig. 32). The driving modules including the articulations are interconnected by means of couplers with the length adjustable to individual anatomic characteristics of the user. The system is fastened to the trunk by means of a clamping ring called a hip belt, which is a kind of a mechanical interface. Motion of the legs is forced by the foot modules connected with the user's feet. Rotary motion at the hip and knee articulations (corresponding to the natural joints of the user) is realized by drive modules, which are custom actuators (consisting of screws and connectors) coupled with pulleys fastened to the articulations. They are built in few sizes adapted to a population.

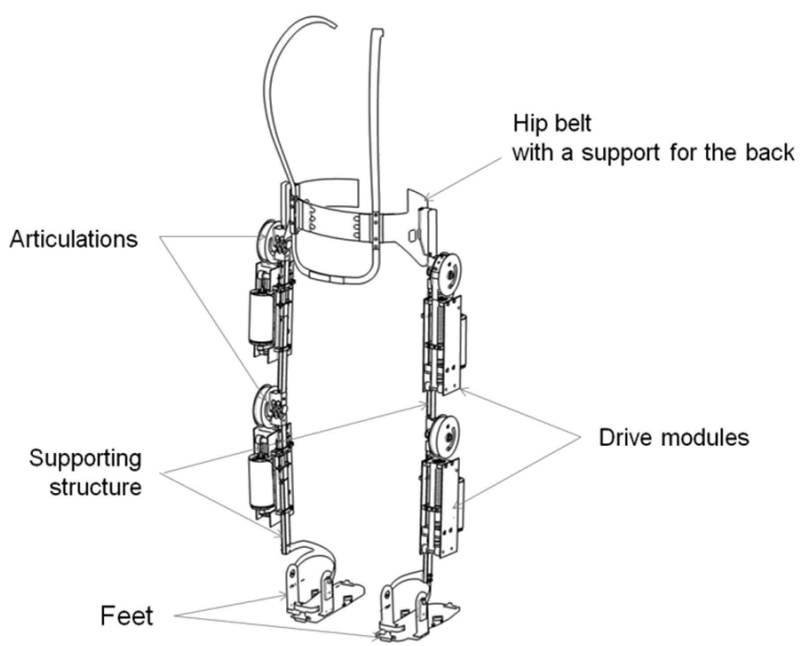

Fig. 32. Scheme of the structure of the designed mechanical subsystem

According to the accepted concept of coupling the driving modules, a precise position adjustment is provided by mechanisms located at the spot where the actuator is coupled with the load-bearing structure. The system that has been designed this way is consistent with the latest trends in prosthetics, which assume creation of series of types of complicated modules (e.g. joints) and coupling them with relatively inexpensive elements adjusted to the measurements of the user. Such approach simplifies personalization of the devices aiding the disabled, and at the same time cuts the related costs.

\section{Summary}

The paper presents the chosen results of the works related to the system for verticalization and aiding the motion of the disabled suffering from a paralysis of the lower limbs. The basic 


\section{Mechatronic system for verticalization and aiding the motion of the disabled}

design works refer to the actuators realizing angular displacements of particular parts of the legs: thigh, shins and feet. At the same time, a safety system has been elaborated. One analyses possibilities of a technical way of aiding the activity of keeping the physical balance of the user and unloading the leg in the phase of the swing. Integration of these systems with the motion system consists, among other things, in checking which of their functions can be realized using the actuators and the measuring systems that have already been created, yet require designing an additional systems. A substantial issue is also deciding at the level of integration of the units controlling particular systems and their software. In this case, a superior criterion is safety of the users.

Acknowledgments. The presented works have been carried out within the ECO-Mobility project No. UDAPOIG.01.03.01-14-154/09-00 supported by the European Union.

\section{REFERENCES}

[1] B. Radziemski, "Elaboration of the design of the dynamic parapodium", MSc Thesis, Warsaw University of Technology, Warszawa, 2007, (in Polish).

[2] Catalog: Rehabilitation Equipment, MISIARZ, Tychy, 2010, (in Polish).

[3] K. Kędzior, M. Pawlikowski, and K. Skalski, "Customised prostheses of human joints and orthosis devices", J. Theoretical and Applied Mechanics 48 (4), 897-915 (2010).

[4] R. Bishop, Mechatronic Systems, Sensors and Actuators. Fundamentals and Modeling, CRC Press, Boca Raton, 2008.

[5] J.L. Pons, Wearable Robots: Biomechatronic Exoskeletons, John Wiley \& Sons, Chichester, 2008.

[6] R. Bogue, "Exoskeletons and robotic prosthetics: a review of recent developments", Industrial Robot: Int. J. 36 (5), 421-427 (2009).

[7] A.M. Dollar and H. Herr, "Lower extremity exoskeletons and active orthoses: challenges and state-of-the-art", IEEE Trans. Robotics 24 (1), 144-158 (2008).

[8] R.J. Farris, H.A. Quintero, and M. Goldfarb, "Preliminary evaluation of a powered lower limb orthosis to aid walking in paraplegic individuals", IEEE Trans. on Neural Systems and Rehabilitation Eng. 19 (6) 652-659 (2011).

[9] S. Mohammed, and Y. Amirat, "Towards intelligent lower limb wearable robots: challenges and perspectives - state of the art", Proc. IEEE Int. Conf. ROBIO 2008 1, 312-317 (2009).

[10] E. Kiel, Drive Solutions. Mechatronics for Production and Logistics, Springer, Berlin, 2008.
[11] M. Gawrysiak, "Mechatronics and mechatronic designing", $B i$ atystok University of Technology Scientific Works 44, CD-ROM (1997).

[12] H. Kawamoto, S. Lee, S. Kanbe, and Y. Sankai , "Power assist method for hal-3 using emg-based feedback controller", Proc. IEEE Int. Conf. Man and Cybernetics Systems 1, 1648-1653 (2003).

[13] Y. Sankai, "Leading edge of cybernics: robot suit HAL", Int. Joint Conf. SICE-ICASE P-1-P2, CD-ROM (2006).

[14] E. Pratt, B. Krupp, C.J. Morse, and S.H. Collins, "The RoboKnee: an exoskeleton for enhancing strength and endurance during walking", Proc. IEEE ICRA'04 3, 2430-2435 (2004).

[15] E. Guizzo and H. Goldstein, "The rise of the body bots", IEEE Spectrum 42 (10), 50-56 (2005).

[16] A. Goffer, Gait-locomotor Apparatus, Patent specification No. EP 1260201B1, 10.12.2008.

[17] Argo Medical Technologies Ltd., Internet site, www.argomedtec.com (access 21.03.2010).

[18] A. Morecki, J. Knapczyk, and K. Kędzior, Theory of Mechanisms and Manipulators. Basics and examples of practical applications, Warsaw, WNT, 2002, (in Polish).

[19] T. Zielińska, Walking Machines. Fundamentals, Designing, Control and Biological Patterns, Scientific Publishing House of PWN, Warsaw, 2003, (in Polish).

[20] J.B. Gonçalves and D.E. Zampieri, "Recurrent neural network approaches for biped walking robot based on zero-moment point criterion", J. Braz. Soc. Mech. Sc. Eng. 25, 69-78 (2003).

[21] F.M. Teixeira Pereira da Silva and J.A. Tenreiro Machado, "Kinematic analysis and modelling of biped locomotion systems", J. Braz. Soc. Mech. Sci. 21 (3), 402-413 (1999).

[22] Otto Bock, Catalog: Helix3D Hip Joint System. Information for Practitioners, Otto Bock HealthCare GmbH, Duderstadt, 2008.

[23] J. Gausemeier, S. Kahl, and S. Pook, "From mechatronics to self-optimizing systems", HNI-Verlagsschriftenreihe 223, 3-34 (2008).

[24] M. Lauder, M. Schlereth, S. Rose, and A. Schürr, "Modeldriven systems engineering: state-of-the-art and research challenges", Bull. Pol. Ac.: Tech. 58 (3), 409-421 (2010).

[25] R. Isermann, Mechatronic Systems - Fundamentals, Springer, Berlin, 2005.

[26] G. Pelz, Mechatronic Systems. Modelling and Simulation with HDLs, John Wiley and Sons Ltd., Chichester, 2003.

[27] T. Zielińska, "Autonomous walking machines - discussion of the prototyping problems", Bull. Pol. Ac.: Tech. 58 (3), 443451 (2010).

[28] B. Mrozek and Z. Mrozek, MATLAB and Simulink. User's Guide, ed. 3, Helion, Gliwice, 2010, (in Polish). 